\title{
Effective action for Superconductors and BCS-Bose crossover
}

\author{
S. De Palo, C. Castellani, C. Di Castro \\ Istituto Nazionale di Fisica della Materia e Dipartimento di Fisica, Universitá La Sapienza, \\ Piazzale A. Moro, 00185 Roma, Italy \\ B. K. Chakraverty \\ Laboratoire d'Études des Propriétés Electroniques des Solides, C.N.R.S.,B.P. 166, 38042 Grenoble Cedex 9, France
}

\begin{abstract}
A standard perturbative expansion around the mean-field solution is used to derive the low-energy effective action for superconductors at $T=0$. Taking into account the density fluctuations at the outset we get the effective action where the density $\rho$ is the conjugated momentum to the phase $\theta$ of the order parameter. In the hydrodynamic regime, the dynamics of the superconductor is described by a time dependent non-linear Schrödinger equation (TDNLS) for the field $\Psi(x)=\sqrt{\frac{\rho}{2}} e^{i \theta}$. The evolution of the density fluctuations in the crossover from weak-coupling (BCS) to strong-coupling (Bose condensation of localized pairs) superconductivity is discussed for the attractive Hubbard model. In the bosonic limit, the TDNLS equation reduces to the the Gross-Pitaevskii equation for the order parameter, as in the standard description of superfluidity. The conditions under which a phase-only action can be derived in the presence of a long-range interaction to describe the physics of the superconductivity of "bad metals" are discussed.
\end{abstract}

PACS numbers: 74.20_z, 74.20.Fg, 67.40.-w

\section{INTRODUCTION}

The description of a superconducting system at low temperatures by an effective action acquired a renewed interest due to the recent attention to vortex dynamicst and to the non-standard behaviorla of the High $T_{c}$ Superconductors (HTCS). The advantage in having an effective theory is well known: the complex microscopic theory is replaced by a simpler one, written in terms of relevant variables only. As an example, near the critical temperature, the equilibriumb and non-equilibrium 4 properties of a superconductor are well described by the Landau-Ginzburg theory that is written in terms of the complex order parameter $\Delta(x)=\left\langle\phi_{\downarrow}(x) \phi_{\uparrow}(x)\right\rangle$.

At zero temperature, the effective theory which allows a minimal description of the low-energy properties of a superconductor (from the Meissner effect to the flux quantization) is written in terms of the phase $\theta(x)$ of the order parameter. Microscopic derivations of such an effective theory are usually carried out by deriving the low temperature effective action in terms of the complex order parameter. This effective action then leads to a wave-efmation for the

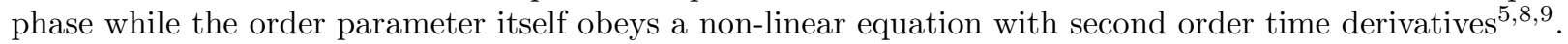

By contrast, the low-energy dynamics of a bosonic superfluid is well described by a Gross-Pitaevskii equation 10 i.e. a time-dependent non-linear Schrödinger equation (TDNLS) for the complex order parameter $\Psi=|\psi| e^{i \theta}$. The continuity equation implied for $|\psi(x)|^{2}$ by the TDNLS description is a continuity equation for the full density which, in this case, can indeed be identified with $|\Psi(x)|^{2}$. For a fermionic superfluid there is instead a non-trivial relation between the density $\rho(x)$ and the order parameter $\Delta(x)$. However, the Gross-Pitaevskii description is expected to hold also for fermions in the strong pairing limit, when the Cooper pairs, that are strongly overlapping in the BCS description, fan be considered as composite bosons. In this limit, the phenomenological effective lagrangian proposed by Feynman 11 , suggesting a description of the superconducting system as a bosonic superfluid, should be recovered.

Recently, Aitchson et al. 12 and M.Stone 13 re-interpreted the wave-like equation for the phase of the order parameter for a BCS superconductor in terms of a non-linear Schrödinger equation. They reconciled the wave-equation with a TDNLS description by re-writing the low-energy effective lagrangian for $\Delta(x)$ in terms of the phase and of the field that plays the role of the conjugated momentum of $\theta(x)$ i.e. the local density $\rho(x)$. The field $\rho(x)$ is however introduced in the theory as an ad hoc field. This procedure needs indeed an identification a posteriori of the "wave-function" obeying the non-linear Schrödinger equation and does not explain the strong coupling limit.

Here we give a microscopic derivation of the zero-temperature low-energy effective action in terms of relevant physical fields, including from the beginning the density $\rho(x)$ together with the complex order parameter $\Delta(x)$ G. Our derivation is quite general. We consider, as usual, small fluctuations of the fields from their mean field values and retain in the effective action only the leading momentum and frequency dependences (hydrodynamic limit).

The effective action is obtained by a standard perturbative procedured with two main differences with respect to previous approaches. First, we decouple the pairing term by means of an Hubbard-Stratonovich (HS) transformation 
in the particle-particle and in the particle-hole channels to include both fluctuations. Since the auxiliary fields introduced by the Hubbard-Stratonovich transformation are not the physical fields, the latter fields are introduced by a further transformation. This makes the low-energy effective lagrangian easily readable and allows for a direct interpretation of the local density as the conjugated momentum of the phase. Furthermore the correct strong pairing limit is straightforward.

The equations of motion supported by this microscopic derivation are the non-linear Schrödinger equation for the variable $\Psi(x)=\sqrt{\frac{\rho(x)}{2}} e^{i \theta(x)}$, in agreement with Ref12 and 13 , and an equation for the modulus of the order parameter $|\Delta(x)|$ relating the local condensate to the local density.

When we consider the BCS-Bose crossover for the attractive Hubbard model, the expression of the lagrangian in terms of the physical fields shows that, on increasing the pairing interaction, the density tends to fluctuate coherently with $|\Delta(x)|^{2}$. In particular, in the low-density limit, the two fields become proportional and the description of lowenergy dynamics by means of two equations of motion becomes redundant. The low-energy dynamics is then described by a Gross-Pitaevskii equation (a TDNLS equation for the complex order parameter $\Delta(x)$ ).

On varioys occasions the crossover between the BCS (weak-coupling) and the Bose (strong-coupling) limit has been studied 14 and the analysis of this problem has acquired particular relevance for the High $\mathrm{T}_{c}$ Superconductors. Indeed, some common characteristics of these compounds (the small value of $k_{F} \xi \simeq 10-20$, compared to the BCS values, and the small carrier density 1 ) can be in favor of a situation close to condensation of localized Cooper pairs, especially in the under-doped HTCS. In this context, the role of the phase fluctuations for this compounds has been analyzed looking at the phase-only action in the presence of a long-range Coulomb force $1 \mathrm{1}, \mathrm{t} \mathrm{d}$. The low-energy action which we obtain, including the density fluctuations together with the fluctuations of the complex order parameter, easily allows to study situations that can deviate from the BCS behavior. Therefore, at the end, we also consider long-range forces in our approach and compare (where possible) our result with the Emery and Kivelson proposal 19 of a phase-only effective action for a description of HTCS as "bad metals".

The paper is organized as follows. The first section is dedicated to the derivation of the low-energy effective lagrangian in terms of the physical fields for a simple continuum model. In section II, we derive the equations of motion and obtain the non-linear Schrödinger equation for the field $\Psi(x)=\sqrt{\frac{\rho(x)}{2}} e^{i \theta(x)}$ In the third section we consider the explicit crossover from weak to strong coupling starting from the attractive Hubbard model. Finally, the effects of a long-range interaction are specifically considered in the last section where the phase-only action is also derived.

\section{THE EFFECTIVE ACTION}

The details of the interaction inducing pairing are not relevant for our purposes. We then consider a very simple model: a fermionic gas, in 3- $d$ dimensions, with a local pairing interaction and an energy cut-off to remove ultraviolet divergences. The "partition function" at $T=0\left(\hbar=k_{B}=1\right)$ for this system is:

$$
\begin{gathered}
\mathcal{Z}=\int \mathcal{D} \phi^{+}(x) \mathcal{D} \phi(x) e^{i S\left(\phi^{+}(x), \phi(x)\right)} \\
S=\sum_{\sigma} \int d^{4} x \phi_{\sigma}^{+}(x)\left[i \partial_{t}+\frac{\nabla^{2}}{2 m}+\mu\right] \phi_{\sigma}(x)-U \int d^{4} x \phi_{\uparrow}^{+}(x) \phi_{\uparrow}(x) \phi_{\downarrow}^{+}(x) \phi_{\downarrow}(x)
\end{gathered}
$$

where $x=(t, \vec{r}), \phi_{\sigma}^{+}(x)$ and $\phi_{\sigma}(x)$ are the Grassmann variables associated to fermions of spin $\sigma, \mu$ is the chemical potential and $U \leq 0$ is the attractive interaction 20 .

To obtain the effective action in terms of relevant variables for the low-energy region, we decouple the interaction term by means of an HS transformation in both the particle-particle and the particle-hole channel, introducing in this way the auxiliary fields associated to the complex order parameter and to the density. The decoupling of the pairing term reads:

$$
\begin{gathered}
\exp \left[-i U \int d^{4} x \phi_{\uparrow}^{+}(x) \phi_{\uparrow}(x) \phi_{\downarrow}^{+}(x) \phi_{\downarrow}(x)\right] \propto \int \mathcal{D} \rho^{H S} \mathcal{D} \rho_{\text {spin }}^{H S} \mathcal{D} \Delta^{H S} \mathcal{D}\left(\Delta^{H S}\right)^{+} \exp \{ \\
-i \alpha \int d^{4} q \int d^{4} k \rho^{H S}(q)\left[\phi_{\uparrow}^{+}(k) \phi_{\uparrow}(k-q)+\phi_{\downarrow}^{+}(k) \phi_{\downarrow}(k-q)\right]+
\end{gathered}
$$




$$
\begin{gathered}
+\alpha \int d^{4} q \int d^{4} k \rho_{\text {spin }}^{H S}(q)\left[\phi_{\uparrow}^{+}(k) \phi_{\uparrow}(k-q)-\phi_{\downarrow}^{+}(k) \phi_{\downarrow}(k-q)\right]+ \\
-i \gamma \int d^{4} k \int d^{4} q\left[\Delta^{H S}(q) \phi_{\uparrow}^{+}(k) \phi_{\downarrow}^{+}(-k-q)+\left(\Delta^{H S}(q)\right)^{+} \phi_{\downarrow}(k) \phi_{\uparrow}(-k-q)\right]+ \\
\left.+i \int d^{4} q \frac{1}{U} \rho^{H S}(q) \rho^{H S}(-q)+\frac{1}{U} \rho_{\text {spin }}^{H S}(q) \rho_{\text {spin }}^{H S}(-q)+\frac{1}{U}\left(\Delta^{H S}(q)\right)^{+} \Delta^{H S}(-q)\right\}
\end{gathered}
$$

where the decoupling in the particle-hole channel has been made following the Hamman prescription: $-U \phi_{\uparrow}^{+}(x) \phi_{\uparrow}(x) \phi_{\downarrow}^{+}(x) \phi_{\downarrow}(x)=-\frac{U}{4}\left[\left(\phi_{\uparrow}^{+}(x) \phi_{\uparrow}(x)+\phi_{\downarrow}^{+}(x) \phi_{\downarrow}(x)\right)^{2}-\left(\phi_{\uparrow}^{+}(x) \phi_{\uparrow}(x)-\phi_{\downarrow}^{+}(x) \phi_{\downarrow}(x)\right)^{2}\right]$ 2 ${ }^{2}$. The relative weights $\alpha$ and $\gamma$ of the p-p and p-h channels are arbitrary but for the "completeness" condition $\alpha^{2}+\gamma^{2}=1$. All the resulting effective actions are equivalent when treated exactly 25 allowing that each channel contributes to the other one at large $q$. However, to have the mean-field results as a starting point, we disregard the "completeness" condition and take $\alpha=\gamma=127$ while introducing a cut-off in the momentum space to prevent over-counting. Indeed, a restricted integration on $q \leq q^{*}$ separates the contributions related to the two channels. Moreover, from now on, we will not consider the field $\rho_{\text {spin }}(x)$ since, in the low-energy limit, spin density fluctuations are completely decoupled from the rest of the system.

In the following we briefly report the main steps of the derivation of the effective action, while its full derivation is reported in the Appendix. As a first step, since the HS fields $\rho^{H S}, \Delta^{H S},\left(\Delta^{H S}\right)^{+}$act as conjugate to the physical fields and coincide with them only at the saddle point level26, we introduce the physical fields by means of a further "Gaussian" transformation of the quadratic terms appearing in (1.2). Secondly, to explicit the dependence on the gradients of the phase $\theta$ \&.12 of order parameter in the action (A1), we make a gauge transformation $\phi(x) \rightarrow \phi(x) e^{i \theta(x) / 2}$.

To obtain the effective action we retain the first and the second order of a standard expansion of the effective bosonic action (A3) which is obtained integrating out the fermionic fields, around the saddle-point solution in the superconducting state. As usual, we assume that the fluctuations of the fields around their saddle point values are small and in particular that the variations of the phase in space and time are slow. The last step consists in integrating out the HS fields in $\mathrm{A} 6$ ).

The effective action in terms of the physical fields reads:

$$
\begin{gathered}
S_{e f f}(\rho,|\Delta|, \theta)=-\int d^{4} x \rho(x)\left[\partial_{t} \theta(x) / 2+\frac{(\nabla \theta)^{2}}{8 m}\right]+ \\
-\int \frac{d^{4} q}{(2 \pi)^{4}}\left\{\delta \rho_{q} \Pi_{\rho \rho}(q) \delta \rho_{-q}+\delta|\Delta|_{q} \Pi_{\Delta \Delta}(q) \delta|\Delta|_{-q}+2 \delta \rho_{q} \Pi_{\rho, \Delta}(q) \delta|\Delta|_{-q}\right\}
\end{gathered}
$$

where

$$
\begin{gathered}
\Pi_{\rho \rho}(q)=\frac{1}{4}\left[\frac{1}{A_{q}-D_{q}}+U-\frac{\left[B_{q}+B_{-q}\right]^{2}}{\left(A_{q}-D_{q}\right)^{2} I_{q}}\right], \\
\Pi_{\Delta \Delta}(q)=-\left[\frac{1}{I_{q}}-U\right], \\
\Pi_{\rho \Delta}(q)=\frac{\left[B_{q}+B_{-q}\right]}{2\left(A_{q}-D_{q}\right) I_{q}}
\end{gathered}
$$

with

$$
I_{q}=\frac{\left[B_{q}+B_{-q}\right]^{2}}{A_{q}-D_{q}}-\left[D_{q}+\frac{1}{2}\left(C_{q}+C_{-q}\right)\right]
$$

The explicit expressions of the above coefficients at $T=0$ are reported in the Appendix.

It is easily checked that eq.(1.3) reproduces the generalized RPA approximation for the response function in the lowfrequency and low-momentum limit 15 . The low-energy and low-momentum excitations of a superconducting systemp described by a microscopic action like (1.1) are density fluctuations: i.e. the Anderson-Bogolubov sound mode22.23. 
In order to describe this collective mode, it is sufficient to consider only the hydrodynamic limit of the effective action (1.3) by taking the $\vec{q}, \omega \rightarrow 0$ limit of the coefficients of the quadratic part in $\rho$ and $|\Delta|$. Notice that at zero temperature the static $(\omega=0, \vec{q} \rightarrow 0)$ and the dynamic limit $(\vec{q}=0, \omega \rightarrow 0)$ of the coefficients in $\sqrt{1.3})$ coincides.

In the BCS limit we recover the usual dispersion for Anderson-Bogolubov mode22.23: $\omega^{2}-v_{F}^{2}\left[1+U N_{0}\right] \mathbf{q}^{2} / 3=0$ where $v_{F}=k_{F} / m$ is the Fermi velocity and $N_{0}$ is the density of states per spin at the Fermi energy. Notice that, in the BCS limit, $\delta \rho(x)$ and $\delta|\Delta|(x)$ are decoupled since $\Pi_{\rho \Delta}^{0}=0$. Indeed, $B_{0} \propto \int d \epsilon N(\epsilon) \epsilon$ vanishes due to the particle-hole symmetry property of the BCS (weak-coupling) theory. Contributions beyond the hydrodynamic limit (i.e. a gradient expansion of the coefficients in the action (1.3)) represent higher order corrections to the sound mode dispersion.

Making a direct comparison with previous derivations 12.13 we find that the density $\rho_{0}$ in front of the phase term has become the full local density $\rho(x)=\delta \rho(x)+\rho_{0}$, while higher order contributions in $\left[\partial_{t} \theta(x) / 2+\frac{(\nabla \theta)^{2}}{8 m}\right]$ are eliminated away under the integration of the HS fields. A direct way to obtain this result is to make an appropriate translation of fields in the action (A3) which is expressed in terms of both the HS fields and the physical fields. We use the following transformation of unitary Jacobian:

$$
\tilde{\rho}^{H S}(x)=\rho^{H S}(x)+\left[\partial_{t} \theta(x) / 2+(\nabla \theta(x))^{2} / 8 m\right]
$$

and rewrite the term $\rho^{H S}(x) \rho(x)$ in the action $(\mathrm{A} 3)$ as $\rho^{H S}(x) \rho(x)=\left\{\tilde{\rho}^{H S}(x)-\left[\partial_{t} \theta(x) / 2+(\nabla \theta(x))^{2} / 8 m\right]\right\} \rho(x)$. The second term of this expression is recognized as the first contribution in the action (1.3). Then the integration over $\tilde{\rho}^{H S}(x)$ and the other HS fields (within the same approximations used previously) results into the phase independent contribution of (1.3). By means of this procedure, we see that the first term of (1.3) is always present in the full action. This result strongly relies on the introduction of the density fluctuations at the outset of our approach and on the use of the physical fields $\rho$ and $\Delta$.

The transformation (1.4) also allows us to see that the dependence of the action on $\partial_{t} \theta(x) / 2$ shown in eq.(1.3) is not restricted to the Gaussian approximation for the HS fields showing that $\rho(x)$ is the proper coefficient of $\partial_{t} \theta(x) / 2$. This approach has to be contrasted to the one of Ref.(11), where the density $\rho(x)$ is derivedphenomenologically by reconstructing $\rho(x)=\rho_{0}+\delta \rho(x)$ via the Galilean invariance from mean field like equations.29

\section{THE LOW-ENERGY EFFECTIVE ACTION: THE NON-LINEAR SCHRÖDINGER EQUATION}

Let us consider, for completeness, the system in the presence of an electro-magnetic field $A \equiv(\varphi(x), \vec{A}(x))$ that we introduce in (1.3) through the minimal substitution on the phase $\partial_{t} \theta / 2 \rightarrow \partial_{t} \theta / 2+e \varphi, \nabla \theta / 2 \rightarrow \nabla \theta / 2-\frac{e}{c} \vec{A}$ and adding the free electromagnetic action. The effective action then reads:

$$
\begin{aligned}
S_{\text {eff }}=- & \int d^{4} x\left\{\rho(x)\left[\partial_{t} \theta(x) / 2+e \varphi(x)+\frac{1}{2 m}\left(\frac{e}{c} \vec{A}(x)-\frac{\nabla \theta(x)}{2}\right)^{2}\right]+\right. \\
+ & {\left[\Pi_{\rho \rho}^{0} \delta \rho(x)^{2}+\Pi_{\Delta \Delta}^{0} \delta|\Delta(x)|^{2}+2 \delta \rho(x) \Pi_{\Delta \rho}^{0} \delta|\Delta(x)|\right]+} \\
& \left.+\frac{1}{8 \pi}\left[\left(\nabla \varphi(x)+\frac{1}{c} \partial_{t} \vec{A}(x)\right)^{2}+(\nabla \wedge \vec{A}(x))^{2}\right]\right\}
\end{aligned}
$$

where we call $\Pi_{\rho \rho}^{0}, \Pi_{\rho \Delta}^{0}, \Pi_{\Delta \Delta}^{0}$ the $q \rightarrow 0$ limit of the coefficients previously defined.

We have already discussed the origin of the first term in (1.3). Because of this term the role of physical density is directly stated: the functional derivative of (2.1) with respect to $\partial_{t} \theta(x)$ gives $-\rho(x) / 2$ and identify half of the physical density as the conjugated momentum to the phase.

This feature suggests to introduce the field $\Psi(x)=\sqrt{\frac{\rho(x)}{2}} e^{i \theta(x)}$ and to re-write an effective action in terms of it. The eq. (2.1) becomes $S_{\text {eff }}=\int d^{4} x \mathcal{L}_{\text {eff }}$ with:

$$
\begin{gathered}
\mathcal{L}_{e f f}=\frac{i}{2}\left[\Psi^{*} \partial_{t} \Psi-\Psi \partial_{t} \Psi^{*}\right]-2 e \varphi \Psi^{*} \Psi-\frac{1}{4 m}\left(i \nabla-\frac{2 e}{c} \vec{A}\right) \Psi^{*}\left(-i \nabla-\frac{2 e}{c} \vec{A}\right) \Psi+ \\
-4 \Pi_{\rho \rho}^{0}\left(\Psi \Psi^{*}-\rho^{0} / 2\right)^{2}-\Pi_{\Delta \Delta}^{0} \delta|\Delta|^{2}-4 \Pi_{\rho \Delta}^{0}\left(\Psi \Psi^{*}-\rho^{0} / 2\right) \delta|\Delta|
\end{gathered}
$$




$$
-\frac{1}{8 \pi}\left[\left(\nabla \varphi+\frac{1}{c} \partial_{t} \vec{A}\right)^{2}+(\nabla \wedge \vec{A})^{2}\right]
$$

Notice that the presence of a ionic background, as usually assumed to conserve the charge neutrality of system, leads to the replacement in (2.2) of $2 e \varphi(x) \Psi^{*} \Psi$ in $e \varphi(x)\left(2 \Psi^{*} \Psi-\rho_{i o n}\right)$. This term vanishes on average since $\rho_{0}=\rho_{i o n}$, but it becomes $e \varphi(x)\left(2 \Psi^{*} \Psi-\rho_{0}\right)$ by retaining the density fluctuations.

The mapping of the low-energy effective action (2.1) into (2.2) holds in the strict hydrodynamic limit. Indeed only the term $\frac{\rho}{8 m}(\nabla \theta)^{2}$ contained in $\frac{1}{4 m} \nabla \Psi^{*} \nabla \Psi$ is present in the lagrangian (2.1), while the remaining term $\frac{1}{32 m \rho}(\nabla \rho)^{2}$ should be evaluated from the gradient expansion of the quadratic contribution $\propto(\delta \rho)^{2}$ in (1.3). However the coefficient of $(\nabla \rho)^{2}$ we obtain from (1.3) is not $\frac{1}{32 m \rho}(\nabla \rho)^{2}$ (for instance, this evaluation at half-filling, for the Hubbard model, gives the same expression $\frac{1}{9 m \rho^{0}} \frac{1}{16}$ we would obtain from expanding the compressibility at low $q$ in the normal phase). Therefore the mapping is reliable only when this difference is irrelevant: i.e. in the low-frequency and low-momentum limit for the the density fluctuations.

The introduction of the field $\Psi(x)$ allow us to derive the equation of motion for $\Psi(x)$ in form of a non-linear Schrödinger equation. By taking the functional derivatives of (2.2) with respect to $\Psi^{*}, \delta|\Delta|$ and $\vec{A}$ we get:

$$
\begin{gathered}
i \partial_{t} \Psi(x)-2 e \varphi(x) \Psi(x)-\frac{1}{4 m}\left(\frac{2 e}{c} \vec{A}(x)+i \nabla\right)^{2} \Psi(x)-\frac{4}{\chi}\left(\Psi(x) \Psi^{*}(x)-\rho^{0} / 2\right) \Psi(x)=0 \\
\delta|\Delta(x)|=-2 \frac{\Pi_{\rho \Delta}^{0}}{\Pi_{\Delta \Delta}^{0}}\left(\Psi(x) \Psi^{*}(x)-\rho^{0} / 2\right) \\
j(x)=\rho(x) \frac{e}{m}\left(\frac{\nabla \theta(x)}{2}-\frac{e}{c} \vec{A}(x)\right)
\end{gathered}
$$

where $\chi$ represents the compressibility given by:

$$
\chi=\frac{1}{2} \frac{\Pi_{\Delta \Delta}^{0}}{\Pi_{\rho \rho}^{0} \Pi_{\Delta \Delta}^{0}-\left(\Pi_{\rho \Delta}^{0}\right)^{2}}=2 \frac{\left(A_{0}-D_{0}\right)\left[1+U\left(D_{0}+C_{0}\right)\right]-4 U B_{0}^{2}}{\left[1+U\left(A_{0}-D_{0}\right)\right]\left[1+U\left(D_{0}+C_{0}\right)\right]-4 U^{2} B_{0}^{2}}
$$

and the current $j(x)$ obeys the Maxwell equation: $\nabla \wedge \vec{B}=\frac{4 \pi}{c} j(x)+\frac{1}{c} \partial_{t} \vec{E}$.

Going back to the variables $\rho(x)$ and $\theta(x)$, the imaginary and real part of the non-linear Schrödinger equation (2.3) are the continuity equation and the equation that links fluctuations in the density to fluctuations in the phase respectively:

$$
\begin{gathered}
\partial_{t} \rho(x)+\nabla\left[\frac{\rho(x)}{m}\left(\frac{\nabla \theta(x)}{2}-\frac{e}{c} \vec{A}(x)\right)\right]=0 \\
\delta \rho(x)=-\chi\left\{\left[\partial_{t} \theta(x) / 2-e \varphi(x)\right]+\frac{1}{2 m}\left(\frac{\nabla \theta(x)}{2}-\frac{e}{c} \vec{A}(x)\right)^{2}\right\}
\end{gathered}
$$

The continuity equation (2.6) is equivalently obtained by setting to zero the functional derivative of (2.1) with respect to $\theta(x)$. The appearance of the continuity equation is indeed due to the fact that the phase is the Goldstone boson field whose associated equation of motion is the charge conservation, provided the electromagnetic response is only given in terms of the phase (as it is the zero temperature case).

As already pointed out, the difference (in general) between the modulus squared $|\Delta|^{2}$ of the order parameter and the density $\rho$ in a superconductor prevents to obtain a description of the dynamics uniquely in terms of a Gross-Pitaevskii equation. In fact, the non-linear Schrödinger equation (2.3) is related to the continuity equation and cannot be extended to describe the condensate $|\Delta|$, but for the bosonic limit which we discuss in the next section. To obtain the dynamics of the condensate we should have made a gradient expansion of the coefficients of the effective action (1.3), at least up to the second derivatives (in time and space) of the modulus $|\Delta|$ to define the coherence length for this field. Being our description limited to the hydrodynamic low-energy regime, the dynamics of the amplitude of the order parameter is missing. Indeed, the local eq.(2.4) should be more properly understood as a static relation, valid at $q=0$, that links the density fluctuations to the order parameter amplitude fluctuations. In conclusion for almost constant $|\Delta|$, the relevant dynamics is described by the non-linear Schrödinger equation for $\Psi(x)$. The coherent length we get for $\Psi(x)$ is linked to the compressibility and is then roughly of the order of inter-particle spacing. 


\section{THE BOSONIC LIMIT: THE ATTRACTIVE HUBBARD MODEL}

We want to analyze the problem of the crossover from standard BCS (weak-coupling) superconductivity to strong coupling superconductivity, where the Cooper pairs are made of couples of strongly bound fermions, by extending the previous approach to the attractive Hubbard model on a lattice 30 . Our aim is to understand the interplay between density and order parameter while increasing the strength of the pairing interaction.

In particular, we want to see how the description of the dynamics of a strong coupling superconductor evolves from the BCS limit to the Gross-Pitaevskii equation and how the role played by the local density is taken by the square of the order parameter amplitude, at least in the low-density limit.

The fermionic action for the Hubbard model is:

$$
S\left(\phi^{+}(x), \phi(x)\right)=\sum_{i, \sigma} \phi_{i, \sigma}^{+}\left(i \partial_{t}+\mu\right) \phi_{i, \sigma}+t \sum_{\langle i j\rangle}\left(\phi_{i, \sigma}^{+} \phi_{j, \sigma}+\phi_{j, \sigma}^{+} \phi_{i, \sigma}\right)-U \sum_{i} \phi_{i, \uparrow}^{+} \phi_{i, \uparrow} \phi_{i, \downarrow}^{+} \phi_{i, \downarrow}
$$

where, as in the previous section, $U \leq 0$, the sum $\langle i j\rangle$ is over the nearest neighbour pairs and the lattice spacing is $a=1$.

In deriving the effective action for the Hubbard model on a lattice two cases are of relevance:

i) the hydrodynamic limit where one can assume small variations $\left|\theta_{i}-\theta_{j}\right| \simeq a\left|\nabla \theta_{i}\right| \ll 1$, for the phase of the order parameter. In this case we follow the same procedure applied in the continuum limit, in particular we expand $-i \operatorname{Tr} \ln \hat{G}^{-1}$ in $(\mathrm{A} 3)$ in powers of $1-\cos \frac{1}{2}\left(\theta_{i}-\theta_{j}\right) \simeq \frac{1}{8}\left(\theta_{i}-\theta_{j}\right)^{2}$ to get the gradient contribution $\left(\nabla \theta_{i}\right)^{2}$.

ii) away from the hydrodynamic limit to allow $\left|\theta_{i}-\theta_{j}\right| \simeq 1$. In this case the differences in the nearest neighbor site phases are not small and the expansion parameter $\left|\theta_{i}-\theta_{j}\right|$ should be substituted by $t /|U|$. Indeed, it is only in the limit $t / U \ll 1$ that one can assign a consistent meaning to the site phase $\theta_{i}[1]$. We shall consider this case in the next section, in connection with the derivation of the phase-only action (4.1).

Here, to analyze the bosonic limit, we hold on the more standard hydrodynamic approach. The derivation is straightforward and the effective low-energy and low-momentum action in terms of the physical fields reads:

$$
\begin{gathered}
S_{e f f}=-\int d x^{4}\left\{\left[\rho(x) \partial_{t} \theta(x) / 2-\frac{\left\langle E_{0}^{K i n}\right\rangle}{16}(\nabla \theta(x))^{2}\right]+\right. \\
\left.+\left[\delta \rho(x) \Pi_{\rho \rho}^{0} \delta \rho(x)+\delta|\Delta(x)| \Pi_{\Delta \Delta}^{0} \delta|\Delta(x)|+2 \delta \rho(x) \Pi_{\Delta \rho}^{0} \delta|\Delta(x)|\right]\right\}
\end{gathered}
$$

where $\Pi_{\rho \rho}^{0}, \Pi_{\rho \Delta}^{0}, \Pi_{\Delta \Delta}^{0}$ are the lattice version of the previously defined coefficients. Obviously, apart from differences due to the lack of Galilean invariance, the effective lagrangians in the continuum (1.3) and in the lattice case (3.2) are similar. Indeed, as far as the explicit time dependence of action is concerned, we obtain the same combination $\rho(x) \partial_{t} \theta(x) / 2$ in both cases. The stiffness is here given in terms of the averaged kinetic energy $\left\langle E_{0}^{K i n}\right\rangle$, consistently with the expression of the diamagnetic contribution for the current, which changes from $-\rho_{0} \frac{e^{2}}{c m}$ into $\frac{e^{2}}{2 c}\left\langle E_{0}^{K i n}\right\rangle$.

In the attractive Hubbard model, except at half-filling where particle-hole symmetry holds, the coefficient, proportional to $B_{0}$, that links fluctuations of the modulus of the order parameter to the density fluctuations is finite and its role is essential for the description of the system in the strong pairing limit.

In order to get informations on the asymptotic (large $|U|$ ) behaviour, we diagonalize the part of the lagrangian (3.2) which is quadratic in the fields $\delta \rho$ and $\delta|\Delta|$. We obtain, in this way, two eigenvectors:

$$
\begin{aligned}
& v_{1}=\frac{\left|\Pi_{\rho \Delta}^{0}\right|}{\left[\left(\Pi_{\rho \Delta}^{0}\right)^{2}+\left(\Pi_{\rho \rho}^{0}-\lambda_{1}\right)^{2}\right]^{1 / 2}} \delta \rho+\frac{\Pi_{\rho \rho}^{0}-\lambda_{1}}{\left[\left(\Pi_{\rho \Delta}^{0}\right)^{2}+\left(\Pi_{\rho \rho}^{0}-\lambda_{1}\right)^{2}\right]^{1 / 2}} \delta|\Delta| \\
& v_{2}=\frac{\left|\Pi_{\rho \Delta}^{0}\right|}{\left[\left(\Pi_{\rho \Delta}^{0}\right)^{2}+\left(\Pi_{\rho \rho}^{0}-\lambda_{1}\right)^{2}\right]^{1 / 2}} \delta \rho+\frac{\lambda_{2}}{\left[\left(\Pi_{\rho \Delta}^{0}\right)^{2}+\left(\Pi_{\rho \rho}^{0}-\lambda_{1}\right)^{2}\right]^{1 / 2}} \delta|\Delta|
\end{aligned}
$$

with eigenvalues:

$$
\lambda_{1,2}=\frac{1}{2}\left\{\left(\Pi_{\rho \rho}^{0}+\Pi_{\Delta \Delta}^{0}\right) \mp\left[\left(\Pi_{\rho \rho}^{0}-\Pi_{\Delta \Delta}^{0}\right)^{2}+4\left(\Pi_{\rho \Delta}^{0}\right)^{2}\right]^{1 / 2}\right\} .
$$

On increasing the interaction, one of the eigenvalues $\left(\lambda_{2}\right)$ diverges like $U^{2}$, while the value of the other $\left(\lambda_{1}\right)$ stays finite. This behaviour implies that the eigenvector corresponding to the diverging eigenvalue, $v_{2}$, has vanishing 
fluctuations $\left\langle v_{2} v_{2}\right\rangle \propto \lambda_{2}^{-1}$. Therefore $\delta \rho$ tends to fluctuate coherently with $\delta|\Delta|$ through the coefficients of the linear combination appearing in $v_{2}$ :

$$
\delta|\Delta| \simeq-\frac{\Pi_{\Delta \Delta}-\lambda_{2}}{\left|\Pi_{\rho \Delta}\right|} \delta \rho
$$

with the ratio $\frac{\Pi_{\Delta \Delta}-\lambda_{2}}{\left|\Pi_{\rho \Delta}\right|}$ staying finite for large $U$, since both $\Pi_{\Delta \Delta}$ and $\Pi_{\rho \Delta}$ diverge like $U^{2}$. The density and the modulus of the order parameter experience the same fluctuations and we expect that they play the same role, so that a description of the low-energy dynamics of system by means of two equations of motion for $\delta \rho$ and $\delta|\Delta|$ is becoming redundant. To clarify further the evolution of fluctuations of $|\Delta|$ we integrate out the density fluctuations and replace $2\left|\Delta_{0}\right| \delta|\Delta| \simeq \delta|\Delta|^{2}$. The resulting effective action is:

$$
\begin{gathered}
S\left(\theta, \delta|\Delta|^{2}\right)=\int d x^{4} \frac{\left\langle E_{0}^{K i n}\right\rangle}{16}(\nabla \theta(x))^{2}-\int d x^{4}\left\{\frac{\rho_{0}}{2} \partial_{t} \theta(x)+\right. \\
\left.-\frac{1}{4 \Delta_{0}} \frac{\Pi_{\rho \Delta}^{0}}{\Pi_{\rho \rho}^{0}}\left(\partial_{t} \theta\left(x_{i}\right)\right) \delta\left|\Delta\left(x_{i}\right)\right|^{2}+\frac{\delta\left|\Delta\left(x_{i}\right)\right|^{2}}{2 \Delta_{0}}\left[\Pi_{\Delta \Delta}^{0}-\frac{\Pi_{\Delta \rho}^{0} \Pi_{\rho \Delta}^{0}}{\Pi_{\rho \rho}^{0}}\right] \frac{\delta|\Delta(x)|^{2}}{2 \Delta_{0}}-\frac{1}{\Pi_{\rho \rho}^{0}}\left(\partial_{t} \theta(x) / 4\right)^{2}\right\}
\end{gathered}
$$

On increasing the interaction, independently from the filling, the term proportional to $\partial_{t} \theta(x) \delta|\Delta(x)|^{2}$ becomes dominant with respect to $\frac{1}{\Pi_{\rho \rho}^{0}}\left(\partial_{t} \theta(x) / 4\right)^{2}$ which only gives negligible corrections to the dispersion of the collective mode. Moreover, in the low-density limit, the coefficient $-\frac{\Pi_{\rho \Delta}^{0}}{4\left|\Delta_{0}\right| \Pi_{\rho \rho}^{0}}$ reaches the unity on increasing the interaction. This behaviour and the fact that the mean field value of $\Delta_{0}^{2}$ is $\rho_{0} / 2$ 22 , allows us to recognize in the effective action (3.4) the contribution $-|\Delta(x)|^{2} \partial_{t} \theta(x)$ where $|\Delta(x)|^{2}=\rho_{0} / 2+\delta|\Delta(x)|^{2}$. In these limits (strong coupling and low-density), the role of the conjugated momentum to the phase is taken by the square of the modulus of the order parameter: indeed, $|\Delta(x)|^{2}$ coincides with $\rho(x) / 2$. Using the same reasoning of the continuum limit we can then get, as a unique equation of motion, a non-linear Schrödinger equation for the field $\Psi=\sqrt{\frac{\rho}{2}} e^{i \theta}$. Since, in this case, $\Psi$ coincides with the order parameter $|\Delta| e^{i \theta}$, the TDNLS results into the Gross-Pitaevskii equation.

In the weak-coupling limit, the delocalized structure of the Cooper pairs does not permit such a description in terms of a Gross-Pitaevskii equation as in a bosonic system. Indeed, we need different equations to govern the dynamics of the density and the modulus of the order parameter. On the contrary, when the pairing interaction is strong enough to form tightly bound Cooper pairs and the density is low enough that there is no overlapping between them, the difference between the square of the modulus of the fermionic order parameter and half of the density becomes negligible and they can be identified: $|\Delta|^{2}=\rho / 2$.

\section{THE PHASE-ONLY ACTION IN THE PRESENCE OF A LONG-RANGE INTERACTION}

We devote this last section to the introduction of a long-range Coulomb interaction $V_{C}(q)=4 \pi e^{2} / \mathbf{q}^{2}$ in the system. In the discussion of the hydrodynamic action in the continuum case we have already implicitly considered a charged system when we introduced the electromagnetic field. This time we pay explicit attention to the effects of the Coulomb interaction on the structure of the resulting effective action when expressed in terms of the phase only.

It has been proposed on several occasion 18 . 19 that a low value of the superfluid density and consequently of the phase stiffness, enhances the role of the phase fluctuations. It is therefore relevant to study the interplay between the phase fluctuations and the low-lying energy modes once the amplitude is kept constant.

To approach this problem let us consider the electron action (3.1) in the presence of the Coulomb interaction: $-\frac{1}{2} \sum_{\sigma, \sigma^{\prime}} \sum_{i, j} V_{C}\left(\overrightarrow{r_{i}}-\vec{r}_{j}\right) \phi_{\sigma}^{+}\left(x_{i}\right) \phi_{\sigma}\left(x_{i}\right) \phi_{\sigma^{\prime}}^{+}\left(x_{j}\right) \phi_{\sigma^{\prime}}\left(x_{j}\right)$. We decouple the long-range interaction in the Hartree channel and then follow the same procedure as before. Finally we obtain the effective action:

$$
\begin{array}{r}
S_{\text {eff }}(\rho,|\Delta|, \theta)=-\int d t \sum_{i} \rho\left(x_{i}\right) \partial_{t} \theta\left(x_{i}\right) / 2+\int d t \sum_{\langle i j\rangle}\left\langle E_{0}^{K i n}\right\rangle / 8\left(1-\cos \left(\theta_{i}-\theta_{j}\right)\right)+ \\
-\int \frac{d \omega}{2 \pi} \sum_{q}\left\{\delta \rho_{q}\left[\Pi_{\rho \rho}(q)+\frac{1}{2} V_{C}(q)\right] \delta \rho_{-q}+\delta|\Delta|_{q} \Pi_{\Delta \Delta}(q) \delta|\Delta|_{-q}+2 \delta \rho_{q} \Pi_{\rho \Delta}(q) \delta|\Delta|_{-q}\right\}
\end{array}
$$

Notice that the coefficient of the quadratic part in the density fluctuations has been modified by the presence of the Coulomb interaction according to RPA prescription. As discussed in Section III, to compare with Ref.(19) and 
Ref.(33) we consider here the discrete form $-\sum_{\langle i j\rangle}\left(1-\cos \left(\theta_{i}-\theta_{j}\right)\right)$ of the gradient term. We recall again that is only in the limit $t /|U| \ll 1$ that we can consistently derive this term. In particular, to get a cosine term which is local in time, we need that the characteristic time over which $\theta_{i}(t)$ varies is much larger than the inverse quasiparticle gap.

By integrating out all the fields but the phase in the action (4.1), we get the phase-only action:

$$
S(\theta)=\frac{1}{8} \int \frac{d \omega}{2 \pi} \sum_{q} \frac{\Pi(q)}{1+V_{C}(q) \Pi(q)} \omega^{2} \theta_{q} \theta_{-q}+\int d t \sum_{\langle i j\rangle}\left\langle E_{0}^{K i n}\right\rangle / 8\left(1-\cos \left(\theta_{i}-\theta_{j}\right)\right)
$$

where

$$
\Pi(q)=\frac{1}{2} \frac{\Pi_{\Delta \Delta}}{\Pi_{\rho \rho} \Pi_{\Delta \Delta}-\Pi_{\rho \Delta}^{2}} .
$$

$\Pi(q)$ reduces to the "short-range" compressibility $\chi$ in the limit $q \rightarrow 0$.

In agreement with Ref.(33), the action is made of two contributions: the quantistic one $\left(\propto \omega^{2} \theta_{q} \theta_{-q}\right)$, whose coefficient is proportional, in the static limit, to the full compressibility in the presence of the Coulomb interaction (and it is therefore vanishing as $\mathbf{q}^{\mathbf{2}}$ ), and the classic term $\left(\propto \cos \left(\theta_{i}-\theta_{j}\right)\right)$ whose coefficient is the stiffness related to the superfluid density $\left(\rho_{s} / m^{*}\right)$.

The above phase-only effective action is obtained for a model with long-range interaction and local pairing interaction without any a priori assumption. At $V_{C}=0$, the action is equivalent to a $x-y$ quantum model in $d+1$ dimensions. On increasing the pairing interaction the stiffness decreases due to the enhancement of the effective mass, while the compressibility increases since we are reaching the bosonic limit, where the chemical potential does not depend on the density 32 .

For $V_{C} \neq 0$, the low-momentum and frequency behaviour of the quantistic term is completely dominated by the divergence of the long-range interaction and the vanishing of the compressibility. In this regime the action becomes:

$$
S \simeq \int \frac{d \omega}{2 \pi} \sum_{q} q^{2} \omega^{2} \frac{1}{32 \pi e^{2}} \theta(q) \theta(-q)+\int d t \sum_{\langle i j\rangle}\left\langle E_{0}^{K i n}\right\rangle / 8\left(1-\cos \left(\theta_{i}-\theta_{j}\right)\right)
$$

In this case, the sound mode corresponding to Anderson-Bogolubov22 23 mode becomes massive with the plasmon frequency given by $\omega_{p}^{2}=4 \pi e^{2} \rho_{0} / m^{*}$ in the continuum and by $\omega_{p}^{2}=-2 \pi e^{2}\left\langle E_{0}^{k i n}\right\rangle$ in the lattice model.

We can extend the analysis to a superconducting system which is coupled to a normal metal through the Coulomb interaction. The density fluctuations of the metal will screen the Coulomb interaction in the Hartree channel and affect the coefficient of the quantistic part. In this case, the coefficient of quantistic part of the effective phase-only action is:

$$
\Pi(q) /\left[1+\frac{V_{C}(q)}{\epsilon(q)} \Pi(q)\right]
$$

where $\epsilon(\omega, \vec{q})=1+V_{C}(q) \Pi_{m}(q)$ and $\Pi_{m}(q)$ is the polarization bubble in the metal. This amounts to replace the Coulomb interaction (in the superconductor) with the effective RPA Coulomb interaction screened by the metal. The low-energy and momentum limit of $(4.4)$ is $\frac{1}{4 e^{2} \pi} \epsilon(\omega, \vec{q}) \mathbf{q}^{2}$. In the "static" region $(\omega \ll|q|)$ the dielectric function is proportional to $1 / \mathbf{q}^{2}$, the quantistic coefficient (4.4) is a constant and does not modify the behaviour of the short-range $\left(V_{C}=0\right)$ action (4.2). On the contrary, in the dynamic limit the dielectric constant is given by $\epsilon \Rightarrow 1+4 \pi i \frac{\sigma(\omega)}{\omega}$ and the quantistic coefficient depends on the $\omega$ behaviour of the conductivity $\sigma(\omega)$.

Assuming that an action like this also holds at finite temperature (with the same expressions for the coefficients) one recovers the Emery and Kivelson proposale 9 for a phase-only action describing HTCS as "bad metals". In this case the onset of the locking of the coherent superconductive phase would be affected by the $\omega$-behaviour of the conductivity. It is tempting to suggest that, near $T_{c}$, the role of the external screening system can also be played by the "normal" component of the superconducting system at finite temperature.

\section{CONCLUDING REMARKS}

The procedure we have followed to derive the effective (hydrodynamic) action for a superconducting system at $T=0$ strongly relies on the introduction of the physical fields $\rho, \Delta, \Delta^{+}$beside the auxiliary fields $\rho^{H S}, \Delta^{H S},\left(\Delta^{H S}\right)^{+}$ introduced to decouple the interaction by means of the Hubbard-Stratonovich transformation. The resulting effective 
action is a proper starting point for selecting relevant features in systems that deviates from the standard BCS superconductors.

The introduction from the outset of the physical density makes it transparent in the action that $\rho(x)$ plays the role of the field conjugated momentum to the phase. As a direct consequence the equation of motion for the field $\Psi(x)=\sqrt{\rho(x) / 2} e^{i \theta(x)}$ has the form of a non-linear Schrödinger equation. However, besides this equation, in general, also the equation of motion for the order parameter amplitude must be considered.

Our procedure allows to follow the evolution of the density fluctuations as the pairing interaction is increased, towards the strong-coupling limit when the Cooper pairs can be considered as true composite bosons. In this case the density fluctuations are identified with the square amplitude fluctuations and in the low-density limit the two fields $\rho(x) / 2$ and $|\Delta(x)|^{2}$ coincide. As a consequence there is no need of two different equations of motion for $\rho(x)$ and $|\Delta(x)|$ as in the BCS case, and the low-energy behaviour can be described by a Gross-Pitaevskii equation for the field $\Psi(x)$ which in this case coincides with the order parameter $\Delta(x)$.

The inclusion of the long-range Coulomb interaction is straightforward and the modifications of the action follows the RPA prescription. Integrating out all the fields but the phase we get the phase-only action in agreement with Ref.(33). In this case a comparison with the proposal of Emery and Kivelson 19 indicates that their effective phaseonly action with the coefficient of the quantistic term proportional to the normal conductivity can be obtained if the superconductor is coupled to a metallic component.

This work has been supported by INFM "Progetto di ricerca avanzata" 1996.

\section{APPENDIX A: THE EFFECTIVE ACTION}

Here we report the derivation of the effective action 1.3 ) in terms of the physical fields $\rho(x),|\Delta(x)|$ and $\theta(x)$, following the steps anticipated in the text.

First, after having decoupled the interaction with the HS fields $\rho^{H S}$ and $\Delta^{H S}$ we introduce the physical fields $\rho$ and $\Delta$ in the action by two Gaussian transformations of the quadratic terms $\left(\rho^{H S}\right)^{2}$ and $\left(\Delta^{H S}\right)^{2}$ appearing in $(1.2)$. In the Nambu formalism, the resulting fermionic-bosonic action, containing both the HS and the physical fields, reads:

$$
\begin{gathered}
S\left(\eta, \eta^{+}, \rho^{H S}, \rho, \Delta^{H S},\left(\Delta^{H S}\right)^{+} \Delta, \Delta^{+}\right)= \\
\int d x^{4}\left\{\eta^{+}(x)\left[i \partial_{t} \sigma_{0}\right] \eta(x)+\eta^{+}(x)\left[\frac{\nabla^{2}}{2 m}+\mu-\rho^{H S}(x)\right] \sigma_{3} \eta(x)+\rho(x) \rho^{H S}(x)+\right. \\
-\eta^{+}(x) \mathcal{R} e \Delta^{H S}(x) \sigma_{1} \eta(x)+\eta^{+}(x) \mathcal{I} m \Delta^{H S}(x) \sigma_{2} \eta(x)+ \\
\left.+\Delta(x)\left(\Delta^{H S}(x)\right)^{+}+\Delta^{+}(x) \Delta^{H S}(x)-\frac{U}{4} \rho^{2}(x)-U|\Delta(x)|^{2}\right\}
\end{gathered}
$$

where $\eta^{+}=\left(\phi_{\uparrow}^{+}, \phi_{\downarrow}\right)$ is the Nambu spinor, $\sigma_{0}$ is the unit matrix and $\sigma_{i}$ is the Pauli matrix vector. Notice that, integrating out $\rho^{H S}(x)$ we get the constraint $\delta\left(\rho(x)-\eta^{+}(x) \sigma_{3} \eta(x)\right)$ that specifies the field $\rho(x)$ as the local density. Analogously, acting on $\Delta^{H S}$, we obtain $\delta\left(\mathcal{R} e \Delta-\frac{1}{2}\left(\phi_{\downarrow} \phi_{\uparrow}+\phi_{\uparrow}^{+} \phi_{\downarrow}^{+}\right)\right.$and $\delta\left(\mathcal{I} m \Delta+\frac{i}{2}\left(\phi_{\downarrow} \phi_{\uparrow}-\phi_{\uparrow}^{+} \phi_{\downarrow}^{+}\right)\right)$.

Next, we get the action directly in terms of the modulus of the physical order parameter and its phase. To this aim, after writing $\Delta=|\Delta| e^{i \theta(x)}$ we make the gauge unitary transformation $\phi(x) \rightarrow \phi(x) e^{i \theta(x) / 2}$ in the action (A1). Then, we remove the dependencies from the phase in the off-diagonal terms and in $\Delta(x)^{H S}|\Delta(x)| e^{-i \theta(x)}+|\Delta(x)| e^{i \theta(x)}\left(\Delta^{H S}(x)\right)^{+}$ by defining the new auxiliary field: $\tilde{\Delta}^{H S}(x)=\Delta^{H S}(x) e^{-i \theta(x)}$. Such procedure is convenient in the low-energy limit, since it explicits the dependence on the gradients of $\theta(x)$.

After these manipulations the action reads:

$$
\begin{aligned}
& S\left(\eta, \eta^{+}, \rho^{H S}, \rho,|\Delta|, \theta, \tilde{\Delta}^{H S},\left(\tilde{\Delta}^{H S}\right)^{+}\right)=\int d x^{4}\left\{\eta^{+}(x)\left[i \partial_{t}+i \frac{\nabla \theta}{2 m} \nabla\right] \sigma_{0} \eta(x)+\right. \\
& +\eta^{+}(x)\left[\frac{\nabla^{2}}{2 m}+\mu-\left(\partial_{t} \theta(x) / 2+\left(\nabla^{\theta}\right)^{2} / 8 m\right)-\rho^{H S}(x)\right] \sigma_{3} \eta(x)+\rho(x) \rho^{H S}(x)+
\end{aligned}
$$




$$
\begin{gathered}
+i \eta^{+}(x) \frac{\nabla^{2} \theta}{4 m} \sigma_{0} \eta(x)+\eta^{+}(x) \mathcal{I} m \tilde{\Delta}^{H S}(x) \sigma_{2} \eta(x)+ \\
\left.-\eta^{+}(x) \mathcal{R} e \tilde{\Delta}^{H S}(x) \sigma_{1} \eta(x)+2|\Delta(x)| \mathcal{R} e \tilde{\Delta}^{H S}(x)-\frac{U}{4} \rho^{2}(x)-U|\Delta(x)|^{2}\right\}
\end{gathered}
$$

By integrating out the fermionic fields we obtain the following bosonic action:

$$
\begin{gathered}
S\left(\theta, \rho^{H S}, \rho,|\Delta|, \tilde{\Delta}^{H S},\left(\tilde{\Delta}^{H S}\right)^{+}\right)=-i \operatorname{Tr} \ln \hat{G}^{-1} \\
+\int d x^{4}\left[\rho(x) \rho^{H S}(x)-\frac{U}{4} \rho^{2}(x)+2|\Delta(x)| \mathcal{R} e \tilde{\Delta}^{H S}(x)-U|\Delta(x)|^{2}\right]
\end{gathered}
$$

Here $\hat{G}$ is the matrix operator representing the one-particle propagator in the presence of the external bosonic fields.

$$
\begin{gathered}
\hat{G}^{-1}=+\left\{\frac{\nabla^{2}}{2 m}+\mu-\rho^{H S}-\left[\partial_{t} \theta / 2+\frac{(\nabla \theta)^{2}}{8 m}\right]\right\} \sigma_{3}+ \\
\left\{\left[i \partial_{t}+i \frac{\nabla \theta}{2 m} \nabla\right]+i \frac{\nabla^{2} \theta}{4 m}\right\} \sigma_{0}-\mathcal{R} e \tilde{\Delta}^{H S}(x) \sigma_{1}-\mathcal{I} m \tilde{\Delta}^{H S}(x) \sigma_{2}
\end{gathered}
$$

We re-write $\hat{G}^{-1}=\hat{G}_{0}^{-1}\left(1-\hat{G}_{0} \Sigma\right)$ by introducing the saddle-point one-particle propagator $\hat{G}_{0}$ and the self-energy matrix $\Sigma$. One has :

$$
\hat{G}_{0}^{-1}=i \partial_{t} \sigma_{0}+\left(\frac{\nabla^{2}}{2 m}+\mu-\rho_{0}^{H S}\right) \sigma_{3}-\Delta_{0}^{H S} \sigma_{1},
$$

which, in the Fourier space, leads to

$$
\hat{G}_{0}=\left(\begin{array}{cc}
G_{0} & F_{0} \\
F_{0} & -G_{0}^{-}
\end{array}\right)=\frac{1}{\omega^{2}-E_{k}^{2}}\left(\begin{array}{cc}
\omega+\epsilon_{k} & \Delta_{0} \\
\Delta_{0} & \omega-\epsilon_{k}
\end{array}\right)
$$

with $G_{0}(\omega, \vec{k})=G_{0}^{-}(-\omega,-\vec{k})$ and $F_{0}(\omega, \vec{k})=F_{0}^{*}(\omega, \vec{k})$. Here $\Delta_{0}^{H S}=U\left|\Delta_{0}\right|, \rho_{0}^{H S}=U \rho_{0} / 2$ are the parameters that extremize the action (A3). The "self-energy" matrix is given by:

$$
\begin{aligned}
\Sigma(x)=\left\{\delta \rho^{H S}(x)\right. & \left.+\left[\partial_{t} \theta(x)+\frac{(\nabla \theta)^{2}}{8 m}\right]\right\} \sigma_{3}-\left[i \frac{\nabla^{2} \theta(x)}{4 m}+i \frac{\nabla \theta(x)}{2 m} \nabla\right] \sigma_{0}+ \\
& +\delta \mathcal{R} e \tilde{\Delta}^{H S}(x) \sigma_{1}-\delta \mathcal{I} m \tilde{\Delta}^{H S}(x) \sigma_{2}
\end{aligned}
$$

We take $\Sigma$ as an expansion parameter, by assuming, as usual, small fluctuations of the fields around their saddle point values and small variations of the phase in space and time, since the dependency of the action in $\theta$ is only through its gradients. We then rewrite $\operatorname{Tr} \ln \hat{G}^{-1}$ in (A3) as $\operatorname{Tr} \ln \hat{G}_{0}^{-1}-\operatorname{Tr} \Sigma_{n} \frac{1}{n}\left(\hat{G}_{0} \Sigma\right)^{n}$ where the factor $\left(\hat{G}_{0} \Sigma\right)^{n}$ is a product of propagators connected into a closed loop by the trace, with $n$ insertions of the fluctuating fields.

To derive the low-energy effective action we retain in (A3) the first and the second order of the expansion in $\left(\hat{G}_{0} \Sigma\right)$ around the superconducting saddle-point:

$$
\begin{gathered}
S_{e f f}\left(\delta \rho, \delta \rho^{H S}, \delta|\Delta|, \theta, \delta \mathcal{R} e \tilde{\Delta}^{H S}\right)=-\int d x^{4} \rho_{0}\left[\partial_{t} \theta(x) / 2+\frac{(\nabla \theta)^{2}}{8 m}\right]+ \\
+\int \frac{d q^{4}}{(2 \pi)^{4}}\left\{-\frac{U}{4} \delta \rho_{q} \delta \rho_{-q}+\delta \rho_{-q}^{H S} \delta \rho_{q}-U \delta|\Delta|_{q} \delta|\Delta|_{-q}+\delta|\Delta|_{q} \delta \mathcal{R} e \tilde{\Delta}_{-q}^{H S}+\right. \\
+\left[\delta \rho_{q}^{H S}+\left(-\frac{i \omega}{2} \theta_{q}+\frac{1}{8 m} \int \frac{d q_{1}^{4}}{(2 \pi)^{4}} \vec{q}_{1}\left(\vec{q}_{1}+\vec{q}\right) \theta_{-q_{1}} \theta_{-q_{1}-q}\right)\right] \\
\cdot[A(q)-D(q)]\left[\delta \rho_{-q}^{H S}+\left(\frac{i \omega}{2} \theta_{-q}+\frac{1}{8 m} \int \frac{d^{4} q_{1}}{(2 \pi)^{4}} \vec{q}_{1}\left(\vec{q}_{1}-\vec{q}\right) \theta_{q_{1}} \theta_{-q_{1}+q}\right)\right]+
\end{gathered}
$$




$$
\begin{gathered}
+\left[\delta \rho_{q}^{H S}+\left(-\frac{i \omega}{2} \theta_{q}+\frac{1}{8 m} \int \frac{d^{4} q_{1}}{(2 \pi)^{4}} \vec{q}_{1}\left(\vec{q}_{1}+\vec{q}\right) \theta_{q_{1}} \theta_{-q_{1}-q}\right)\right]\left[B_{q}+B_{-q}\right] \delta \mathcal{R} e \tilde{\Delta}_{-q}^{H S} \\
-2 i\left[\delta \rho_{q}^{H S}+\left(-\frac{i \omega}{2} \theta_{q}+\frac{1}{8 m} \int \frac{d^{4} q_{1}}{(2 \pi)^{4}} \vec{q}_{1}\left(\vec{q}_{1}+\vec{q}\right) \theta_{q_{1}} \theta_{-q_{1}-q}\right)\right]\left[B_{q}-B_{-q}\right] \delta \mathcal{I} m \tilde{\Delta}_{-q}^{H S} \\
+i \delta \mathcal{I} m \tilde{\Delta}_{q}^{H S}\left[C_{q}-C_{-q}\right] \delta \mathcal{R} e \tilde{\Delta}_{-q}^{H S}-\delta \mathcal{I} m \tilde{\Delta}_{q}^{H S}\left[D_{q}-\frac{1}{2}\left(C_{q}+C_{-q}\right)\right] \delta \mathcal{I} m \tilde{\Delta}_{-q}^{H S} \\
\left.+\delta \mathcal{R} e \tilde{\Delta}_{q}^{H S}\left[D_{q}+\frac{1}{2}\left(C_{q}+C_{-q}\right)\right] \delta \mathcal{R} e \tilde{\Delta}_{-q}^{H S}\right\}
\end{gathered}
$$

where the coefficients 28 are:

$$
\begin{aligned}
& A(q)=\frac{i}{2} \int \frac{d k^{4}}{(2 \pi)^{4}}\left[G_{0}(k+q) G_{0}(k)+G_{0}^{-}(k+q) G_{0}^{-}(k)\right] \\
& =-\frac{1}{2} \int \frac{d k^{3}}{(2 \pi)^{3}} \frac{\epsilon_{+} \epsilon_{-}-E_{+} E_{-}}{E_{+} E_{-}} \frac{E_{+}+E_{-}}{\left(E_{+}+E_{-}\right)^{2}-\omega^{2}} \\
& B(q)=\frac{i}{2} \int \frac{d k^{4}}{(2 \pi)^{4}}\left[F_{0}(k+q) G_{0}(k)+G_{0}^{-}(k+q) F_{0}(k)\right] \\
& =-\frac{\Delta_{0}}{4} \int \frac{d k^{3}}{(2 \pi)^{3}}\left[\frac{\epsilon_{+}+\epsilon_{-}}{E_{+} E_{-}}+\frac{\omega}{E_{+} E_{-}}\right] \frac{E_{+}+E_{-}}{\left(E_{+}+E_{-}\right)^{2}-\omega^{2}} \\
& C(q)=-i \int \frac{d k^{4}}{(2 \pi)^{4}} G_{0}(k+q) G_{0}^{-}(k) \\
& =\frac{1}{2} \int \frac{d k^{3}}{(2 \pi)^{3}}\left[\frac{E_{+} E_{-}+\epsilon_{+} \epsilon_{-}}{E_{+} E_{-}}\left(E_{+}+E_{-}\right)+\omega\left(\frac{\epsilon_{+}}{E_{+}}+\frac{\epsilon_{-}}{E_{-}}\right)\right] \frac{1}{\left(E_{+}+E_{-}\right)^{2}-\omega^{2}} \\
& D(q)=i \int \frac{d k^{4}}{(2 \pi)^{4}} F_{0}(k+q) F_{0}(k) \\
& =-\frac{\Delta_{0}^{2}}{2} \int \frac{d k^{3}}{(2 \pi)^{3}} \frac{1}{E_{+} E_{-}} \frac{E_{+}+E_{-}}{\left(E_{+}+E_{-}\right)^{2}-\omega^{2}}
\end{aligned}
$$

and, in our notation:

$$
\begin{gathered}
E_{ \pm}=\left[\epsilon_{ \pm}^{2}+\left|\Delta_{0}^{H S}\right|^{2}\right]^{1 / 2} \\
\epsilon_{+}=(k+q)^{2} / 2 m-\mu-\rho_{0}^{H S} \text { and } \epsilon_{-}=k^{2} / 2 m-\mu-\rho_{0}^{H S}
\end{gathered}
$$

Notice that in the $q \rightarrow 0$ limit one gets $C_{0}+D_{0}=-\frac{1}{U}-\left(A_{0}-D_{0}\right)$. In the BCS limit one recovers: $A_{q}-D_{q} \simeq$ $\frac{m k_{F}}{2 \pi^{2}}=N_{0}$ and $B_{0}=0$; while in the strong coupling limit one obtains $A_{0}-D_{0} \simeq-\frac{4 \Delta_{0}^{2}}{U}$ and $B_{0}=-\left(1-\rho_{0}\right) \Delta_{0} \frac{1}{U}$.

Since we are interested in the low-energy regime of the system in the derivation of (A6) we have neglected contributions that gives corrections that go beyond this regime. We have indeed neglected terms associated to the expansion of $-\left[i \frac{\nabla^{2} \theta(x)}{4 m}+i \frac{\nabla \theta(x)}{2 m} \nabla\right] \sigma_{0}$ whose contribution is proportional 
to $\int d^{4} q \frac{\vec{q}}{2 m} \theta_{q} \int d^{4} k\left[(\vec{k}+\vec{q}) G_{0}(k+q) \vec{k} G_{0}(k)+(\vec{k}+\vec{q}) F_{0}(k+q) \vec{k} F_{0}(k)\right] \frac{\vec{q}}{2 m} \theta_{-q} . \quad$ The coefficient of this term $\left(\int d^{4} k\left[(\vec{k}+\vec{q}) G_{0}(k+q) \vec{k} G_{0}(k)+(\vec{k}+\vec{q}) F_{0}(k+q) \vec{k} F_{0}(k)\right]\right)$ represents the paramagnetic current and it vanishes in the static limit at $T=0$, while, at finite temperature, it gives the contribution that, due to the presence of the normal component of the superfluid, reduces the stiffness. At zero temperature the first contribution to the action coming from this term is of order $\left(\nabla^{2} \theta\right)^{2}$ and then negligible in the low-energy limit. This also shows that the expansion of $-\left[i \frac{\nabla^{2} \theta(x)}{4 m}+i \frac{\nabla \theta(x)}{2 m} \nabla\right] \sigma_{0}$ does not generate time derivatives of $\theta$. This expansion can only give time derivatives of $\left(\nabla^{2} \theta\right)$, the first non vanishing term being $\left(\nabla^{2} \dot{\theta}\right)^{2}$.

The last step consists in integrating out the HS fields to get the effective action (1.3). Since only the first and the second order terms in the expansion in powers of $\left(\hat{G}_{0} \Sigma\right)$ were retained, the action A3) is Gaussian in the auxiliary fields even though it contains higher order corrections in the phase fields. We can therefore straightforwardly integrate the HS fields to get the action in terms of the physical fields reported in (1.3).

Notice that in this integration we can ignore contributions coming from the coupling of $\delta \mathcal{I} m \tilde{\Delta}^{H S}$ with the other HS fields. The corrections coming from this coupling to the coefficients of the effective action (1.3) represent higher order corrections to the hydrodynamic limit.

We devote the remaining few comments on Galilean invariance. Notice that by making explicit the dependence of the action (A2) on the phase of the order parameter, the Galilean invariance of the action becomes transparent. A Galilean transformation is described by the following change of variables:

$$
\begin{array}{ll}
\vec{r}^{\prime}=\vec{r}-\vec{v} t & \frac{d}{d t^{\prime}}=\partial_{t}+\vec{v} \cdot \nabla \\
t^{\prime}=t & \nabla^{\prime}=\nabla
\end{array}
$$

where $\vec{v}$ is the velocity of the moving frame. Under a Galilean transformation $\Delta(x) \Rightarrow \Delta(x) e^{-2 i\left[m \vec{v} \cdot \vec{r}-m v^{2} t / 2\right]}$ and therefore the phase changes into:

$$
\theta^{\prime}\left(x^{\prime}\right)=\theta(x)-2 m \vec{v} \cdot \vec{r}+m v^{2} t
$$

In the action $(\mathrm{A} 2)$ we can individuate two Galilean invariant terms:

i) $\left[i \partial_{t}+i \frac{\nabla \theta}{2 m} \nabla\right]$, that can be interpreted as the "covariant" derivative,

ii) $\left[\partial_{t} \theta(x) / 2+(\nabla \theta)^{2} / 8 m\right]$, where $\partial_{t} \theta(x) / 2$ is the local chemical potential shift while $(\nabla \theta)^{2} / 8 m$ will give rise to the superfluid contribution to the kinetic energy of the system, once we define the superfluid velocity $v_{s}=(\nabla \theta) / 2 \mathrm{~m}$.

Notice that, deriving the effective action (A6) we have spoiled the "covariant" derivative $\left[i \partial_{t}+i \frac{\nabla \theta}{2 m} \nabla\right]$ contained in the one-particle "propagator" (A4). As a consequence, for a given order in the perturbation theory, the Galilean invariance for the effective action can only be recovered taking into account appropriate terms from higher order contributions 8 , 22 .

${ }^{1}$ E.B. Sonin, Rev. Mod. Phys. 59, 87 (1987); Ji-Min Duan, Phys. Rev. B 48, 333 (1993); R. Iengo and G.Jug, Phys. Rev. B 52, 7537 (1995); D. J. Thouless et al., Phys. Rev. Lett. 76, 3758 (1996); X.-M. Zhu et al. Phys. Rev. Lett. 77, 562 (1996) X.-M. Zhu et al. Phys. Rev. Lett. 78, 122 (1997)

2 See for example the proceedings of the Conferences "Materials and Mechanisms of High Temperature Superconductivity I-V" in Physica C 185-9, (1991); Physica C 235-240, (1994); Physica C 282-287, (1997)

${ }^{3}$ V. L. Ginzburg and L. D. Landau, Zh. Eksperim.i Teor. Fiz. 20, 1064 (1950)

${ }^{4}$ M. J. Stephen and H. Suhl, Phys. Rev. Lett. 13, 797 (1964); A. Schmid, Phys. Konders. Materie, 5, 302 (1966); C. Di Castro, W. Young, Il Nuovo Cimento 62, 273 (1969);

${ }^{5}$ E. Abrahams and T. Tsuneto, Phys. Rev. 152, 416 (1966)

${ }^{6}$ For a discussion of the equation of motion for the density field $\rho(x)$ in addition to the order parameter to complete the time dependent Landau-Ginzburg description near $T_{c}$ see for instance Di Castro and Young in Ref. (4).

${ }^{7}$ H. Kleinert, Forts. Phys. 26, 565-671 (1978)

${ }^{8}$ M. P. Kemoklidze and L. P. Pitaevskii, Sov. Phys JETP 22, 160 (1967)

${ }^{9}$ H. T. C. Stoof, Phys. Rev. B 47, 7979 (1993)

${ }^{10}$ E. P. Gross, Il Nuovo Cimento, 20, 454 (1961); L. P. Pitaevskii, Sov. Phys JETP 12, 155 (1961) 
${ }^{11}$ R. P. Feynman, Statistical Mechanics, Reading, Massachusetts, 1972

${ }^{12}$ Ian J.R. Aitchison, Ping Ao, David J. Thouless, and X.M. Zhu Phys. Rev. B 51, 6531 (1995).

${ }^{13}$ M. Stone, Int. J. Mod. Phys. B 9, 1359 (1995)

${ }^{14}$ M. Randeria Crossover from BCS Theory to Bose-Einstein Condensation: a Review, "Bose-Einstein Condensation", (A. Griffin, D. Snoke and S. Stringari, Cambridge University Press, 1994) and reference therein; J. Engelbrecht et al., Phys. Rev. B 55, $15153(1997)$

${ }^{15}$ R. Micnas, J. Ranninger, S. Robasziewicz, Rev. Mod. Phys, 62, 113 (1990); T. Kostyrko and R. Micnas Phys. Rev. B 46, 11025 (1992); T. Kostyrko and J.Ranninger, Phys. Rev. B 54, 1 (1996)

${ }^{16}$ R. Haussmann, Z. Phys. B 91, 291 (1993); F. Pistolesi and G.C. Strinati, Phys. Rev. B 49, 6356 (1994); R. Haussmann, Phys. Rev. B 49, 12975 (1994); F. Pistolesi and G.C. Strinati, Phys. Rev. B 53, 15168 (1996)

17 Y.L. Uemura et al., Nature 364, 605 (1993); Ch. Niedermayer et al., Phys. Rev. Lett. 71, 1764 (1993); M. Weber et al., Phys. Rev. B 48, 13022 (1990); J. P. Franck et al., Phys. Rev. Lett. 71, 283 (1993); D. N. Basov et al., Phys. Rev. Lett. 77, $598(1995)$

18 B. K. Chakraverty, A. Taraphder and M. Avignon, Physica C 235-240, 2323 (1994).

${ }^{19}$ V. J. Emery and S. A. Kivelson, Phys. Rev. Lett. 74, 3253 (1995)

${ }^{20}$ We shall work in the real time framework of Ref.(7) for a more direct comparison with Ref.(12) even though the imaginary time formalism would have been more transparent in dealing with the functional integrals.

${ }^{21}$ Y. Nambu, Phys. Rev. 117, 648 (1960)

${ }^{22}$ N. N. Bogolubov, Sov. JETP 34, 41, 51 (1958)

${ }^{23}$ P.W. Anderson, Phys. Rev. 112, 1900 (1958)

${ }^{24}$ D. R. Hammann, Phys. Rev. Lett. 23, 95 (1969)

${ }^{25}$ See C. Castellani and C. Di Castro, Phys. Lett. 70, 37 (1979) and references therein.

${ }^{26}$ It is indeed known that the propagators of the HS fields are different from the propagators of the physical ones. As an example, the density-density propagator differs from the analogous auxiliary field propagator by a constant contribution (apart from a multiplicative factor that could be adsorbed in the definition of $\rho^{H S}$ ):

$$
\langle\rho(x) \rho(y)\rangle=\frac{4}{U^{2}}\left\langle\rho^{H S}(x) \rho^{H S}(y)\right\rangle-\frac{2}{U} \delta(x-y) .
$$

${ }^{27}$ Indeed, the choice $\alpha=\gamma=1$ gives the standard mean field results as saddle point solutions and, at the same time, guarantees that the Gaussian action reproduces the RPA approximation.

${ }^{28}$ A. J. Leggett, Phys. Rev. 147, 119 (1966)

${ }^{29}$ Notice however that the lack of Galilean invariance of the mean field equations derived for instance from the time dependent Landau-Ginzburg theory is not an intrinsic problem of the time dependent Landau-Ginzburg theory but of the mean-field approximation in the hydrodynamic regime.

${ }^{30}$ The introduction of a lattice is the simplest way to introduce an "ultraviolet" cut-off to regularize the strong coupling limit.

${ }^{31}$ We thank M. Randeria for making clear to us this important issue.

32 P. Nozieres and S. Schmitt-Rink, J. Low Temp. Phys. 59, 195 (1985)

${ }^{33}$ T. V. Ramakrishnan, Phys. Scripta, T 27, 24 (1989) 